\title{
Extracranial metastasis of gliobastoma: Three illustrative cases and current review of the molecular pathology and management strategies
}

\author{
ABHISHEK RAY ${ }^{1}$, SUNIL MANJILA ${ }^{1}$, ALIA M. HDEIB ${ }^{1}$, ARCHANA RADHAKRISHNAN ${ }^{2}$, \\ CHARLES J. NOCK ${ }^{2}$, MARK L. COHEN $^{3}$ and ANDREW E. SLOAN ${ }^{1,3}$
}

${ }^{1}$ Brain Tumor and Neuro-Oncology Center and Department of Neurological Surgery, Case Western Reserve University
School of Medicine; Departments of ${ }^{2}$ Oncology and ${ }^{3}$ Pathology, University Hospitals Case Medical Center,
Seidman Cancer Center and Case Comprehensive Cancer Center, Cleveland, OH 44106, USA

Received November 13, 2014; Accepted January 8, 2015

DOI: $10.3892 / \mathrm{mco} .2015 .494$

\begin{abstract}
Glioblastoma (GBM) is the most common and the most malignant primary brain tumor in adults, accounting for $\sim 12-15 \%$ of all intracranial neoplasms. Despite advances in surgical, medical and radiation therapies, the mortality of GBM remains high, with a median survival ranging between 40 and 70 weeks. Similar to other primary brain tumors, the extracranial metastasis of GBM is extremely rare, occurring in $<2 \%$ of patients. To demonstrate the clinical characteristics of this rare tumor, we herein present three cases of extracranial GBM metastasis: One to the lungs, which represents the longest reported survival of lung metastases from GBM to date; the second to the soft tissue of the posterior neck; and the third to the lumbar intradural space. Unlike tumors elsewhere, there are unique barriers in the brain that prevent the hematogenous and lymphatic spread of intracranial tumors, such as the dura mater and the thickened basement membrane of the blood vessels. In addition, central nervous system tumor cells lack extracellular matrix proteins required to invade surrounding connective tissue, a prerequisite for tumor dissemination. In this study, we aimed to investigate the different possible mechanisms underlying the extracranial metastasis of GBM and determine the biomolecular and genetic characteristics differentiating GBMs that metastasize from those that do not. We also reviewed the role of systemic chemotherapy and bevacizumab in the treatment of disseminated GBMs. Early identification and differentiation of these tumors may enable
\end{abstract}

Correspondence to: Dr Andrew E. Sloan, Brain Tumor and Neuro-Oncology Center and Department of Neurological Surgery, Case Western Reserve University School of Medicine, University Hospitals Case Medical Center and Seidman Cancer Center, 11100 Euclid Avenue, Hanna House 524, Cleveland, OH 44106, USA

E-mail: andrew.sloan@uhhospitals.org

Key words: glioblastoma, metastasis, bevacizumab, temozolomide, glial fibrillary acidic protein patients to benefit from surgical resection, radiation and combination chemotherapy prior to developing other comorbidities from metastatic disease, which may translate into prolonged survival with an acceptable quality of life.

\section{Introduction}

Glioblastoma (GBM) is the most common primary brain tumor in adults, accounting for $\sim 12-15 \%$ of all intracranial neoplasms (1). Despite advances in surgical, medical and radiation therapies, the mortality of GBM remains high, with a median survival ranging between 40 and 70 weeks (2). Like other primary brain tumors, the extracranial metastasis of GBM is extremely rare, occurring in $<2 \%$ of all GBMs (3-8). A recently published meta-analysis of 88 cases of extracranial GBM between 1928 and 2009 demonstrated that the prognosis is particularly poor in this subset of patients, with a median survival from metastasis to death of 1.5 months; however, there has been a progressive prolongation of this interval of 0.7 months per decade between 1940 and 2000 (9). Patients treated with surgery, radiation, chemotherapy and cerebrospinal fluid (CSF) shunting exhibit the longest average survival interval from metastasis to death.

Unique barriers in the brain, including the blood-brain barrier, thickened basement membrane of blood vessels and thickened dura mater, lack of extracellular matrix and other characteristics unique to GBM, prevent hematogenous and lymphatic spread. The majority of metastases reportedly occur either through leptomeningeal or intramedullary dissemination to the spinal cord or following a breach in the abovementioned barriers, e.g., through craniotomy or intraventricular shunt placement. In the latter, metastasis is considered to occur by direct spread or tumor seeding. Metastases of malignant gliomas to the soft tissue, muscle, or skin, are even more rare, with only nine cases reported in the literature (10). We herein present three cases of GBM metastasis: one to the lung, one to the soft tissue of the posterior neck and one to the lumbar intradural space (Table I). Of note, our first case represents the longest survival interval from diagnosis of GBM lung metastases to death reported in the literature thus far. We also 
Table I. Summary of case reports.

\begin{tabular}{|c|c|c|c|c|c|c|c|c|}
\hline Case & $\begin{array}{l}\text { Age/ } \\
\text { gender }\end{array}$ & $\begin{array}{c}\text { Site of } \\
\text { metastasis }\end{array}$ & $\begin{array}{l}\text { Time from } \\
\text { Dx to Met } \\
\text { (months) }\end{array}$ & $\begin{array}{l}\text { Time from } \\
\text { Dx to death } \\
\text { (months) }\end{array}$ & $\begin{array}{l}\text { Time from } \\
\text { Met to death } \\
\text { (months) }\end{array}$ & Surgery & Chemotherapy & Radiation \\
\hline 1 & $29 / \mathrm{F}$ & Lung & 22 & 35 & 13 & $\begin{array}{l}\text { Gross total resection } \\
2 \text { repeat resections, } \\
\text { vp shunt }\end{array}$ & $\begin{array}{l}\text { Temozolomide, } \\
\text { bevacizumab, } \\
\text { carboplatin }\end{array}$ & $\begin{array}{l}60 \mathrm{~Gy}, \\
\text { re-irradiation } \\
38.5 \mathrm{~Gy}\end{array}$ \\
\hline 2 & 29/M & $\begin{array}{l}\text { Soft tissue of } \\
\text { posterior neck }\end{array}$ & 13 & 17 & 4 & $\begin{array}{l}\text { Subtotal resection, } \\
\text { repeat resection }\end{array}$ & $\begin{array}{l}\text { Procarbazine, } \\
\text { lomustine, } \\
\text { vincristine }\end{array}$ & 60 Gy \\
\hline 3 & $62 / \mathrm{M}$ & $\begin{array}{l}\text { Spine and } \\
\text { sacral nerve } \\
\text { root }\end{array}$ & 0 & 6 & 6 & None & None & $\begin{array}{l}46 \text { Gy whole-brain, } \\
46 \text { Gy C-spine, } \\
5,000 \text { cGy sacral }\end{array}$ \\
\hline
\end{tabular}

Dx, diagnosis; Met, metastasis; F, female; vp, ventriculoperitoneal; M, male; C-spine, cervical spine.

reviewed the relevant literature in an attempt to delineate the molecular and genetic basis for GBM metastasis and the treatment strategies for this aggressive disease.

\section{Case reports}

Case 1. A 29-year-old woman presented with a 6-month history of progressively worsening headaches, vision loss and cachexia. The magnetic resonance imaging (MRI) revealed a complex $7-\mathrm{cm}$ mass in the right posterior temporal and parietal lobes (Fig. 1) with multiple intramural cysts, exhibiting extensive septal and nodular enhancement within the lesion. There was also perilesional edema with a $5-\mathrm{mm}$ midline shift to the left side. The patient underwent gross total resection followed by adjuvant chemotherapy with temozolomide and radiation therapy (60 Gy). The patient then was continued on maintenance temozolomide and exhibited significant resolution of her symptoms. The maintenance therapy was interrupted for 3 months due to the development of cytopenias and secondary infections. Approximately 14 months after the initial presentation, the patient complained of increasing headaches and had new-onset seizures. The cranial MRI revealed abnormal enhancing areas involving the right lateral aspect of the tentorium cerebelli and right transverse sinus-sigmoid sinus junction, consistent with tumor recurrence. The patient was re-irradiated (38.5 Gy) and exhibited an initial resolution of the headaches, which recurred 3 months later. Repeat MRI revealed a nodular $3.2 \times 2.6-\mathrm{cm}$ mass along the right lateral aspect of the tentorium, with abnormal leptomeningeal enhancement adjacent to the mass and of the cranial nerves VIII and IX. On biopsy, the lesion proved to be radiation necrosis rather than tumor. However, a preoperative chest X-ray revealed asymptomatic bilateral, multiple rounded hyperdensities in the lungs, suggestive of pulmonary metastases (Fig. 1), which measured $3.4 \times 4.2 \mathrm{~cm}$ on computed tomography (CT). The biopsy was consistent with metastatic GBM (Fig. 2). Immunohistochemical staining for S100 protein and glial fibrillary acidic protein (GFAP) was positive in the tumor cells. The patient presented 4 months later with recurrent headaches as well as gait alterations. The MRI showed hydrocephalus and a ventriculoperitoneal shunt was placed. At 24 months after the initial diagnosis, systemic chemotherapy with bevacizumab and carboplatin was reinitiated for progressive disease and continued for 6 months, followed by single-agent bevaciszumab when a restaging cranial MRI demonstrated progressive disease with extension of the tumor into the right posterior fossa. At that time, the patient underwent a third resection. Despite an initial improvement in the headaches and ataxia, the patient subsequently exhibited a significant decline in function, generalized weakness and, at 34 months, entered hospice and declined further care. The patient succumbed to the disease 1 month later, 35 months after the initial diagnosis.

Case 2. A 29-year-old man presented with a 2-week history of confusion, lethargy and a decline in the ability to perform his daily activities. On examination, the patient was oriented to person and place. There were no lateralizing focal neurological deficits. A CT and MRI of the head revealed a large left frontal lobe mass measuring $6.5 \times 7.5 \mathrm{~cm}$, with areas of calcification and surrounding edema, exerting a mass effect on the ipsilateral lateral ventricle. The mass exhibited diffuse heterogeneous enhancement and small areas of cystic components, along with a small hemorrhage within the mass. The patient underwent left frontal craniotomy for subtotal tumor resection. On pathological examination of the surgical specimen, the tumor was diagnosed as anaplastic oligodendroglioma. Immunohistochemical staining for GFAP was strongly positive in a significant proportion of the tumor cells. The patient improved clinically and became more lucid. Postoperatively, the patient received radiation therapy (60 Gy) and chemotherapy with procarbazine, lomustine and vincristine for 6 weeks. Three months after the initial surgery, the patient presented with a symptomatic recurrence of his left frontal lobe mass and underwent another resection. Compared to the previous specimen, there was an overwhelming predominance of neoplastic astrocytes, upgrading the tumor to GBM. At 13 months from the initial diagnosis, the patient presented with a progressively enlarging, pain-free, right posterior neck mass. 

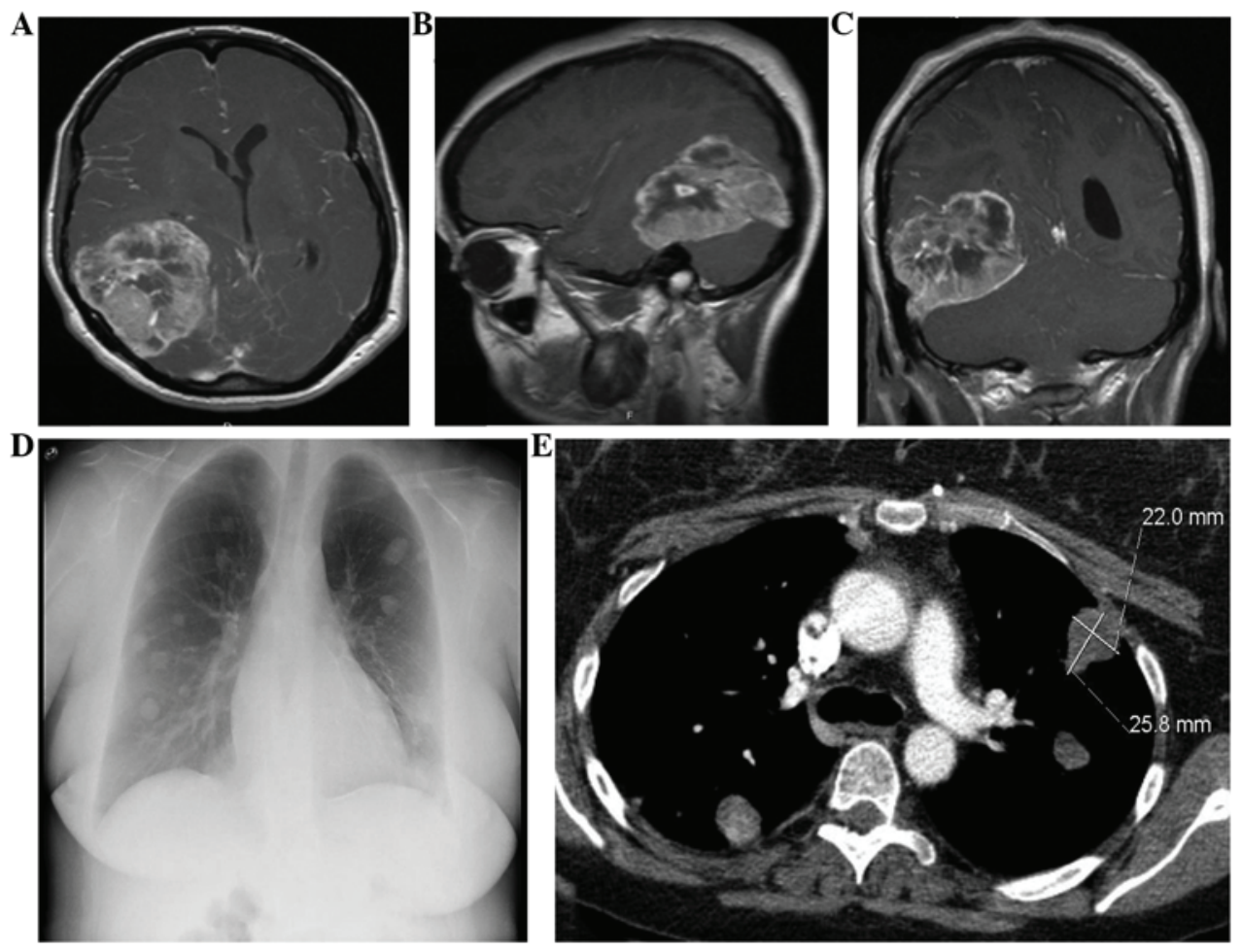

Figure 1. Case 1: Contrast-enhanced magnetic resonance imaging (A) axial, (B) sagittal and (C) coronal sequences shows primary intracranial glioblastoma. Chest (D) X-ray and (E) contrast-enhanced computed tomography reveal metastatic glioblastoma to the lungs.

Following a needle biopsy and histopathological examination, the neck mass exhibited similar characteristics to the central nervous system (CNS) tumor and was diagnosed as metastatic GBM (Fig. 2). Immunohistochemical stains with antibodies directed against S100 protein and GFAP were reactive with the tumor cells. Synaptophysin immunostaining, which was initially non-reactive, was found to be positive, possibly representing a more primitive clone with divergent differentiation, similar to that seen in primitive neuroectodermal tumors. The patient succumbed to the disease 4 months later, 17 months after the initial diagnosis.

Case 3. A 62-year-old man presented with right-sided low back pain and pain in the buttocks, which occasionally radiated down the lateral aspects of his right leg. The patient did complain of occasional frontal headaches over the past several years; however, the neurological examination was unremarkable. An MRI of the lumbar spine revealed a homogenously enhancing mass in the L5-S1 region to the right of the midline, measuring $9 \times 10 \times 15 \mathrm{~mm}$ and exerting a moderate mass effect, pushing the thecal sac to the left (Fig. 3). The imaging characteristics were considered to be suggestive of a right S1 schwannoma. The electromyogram was consistent with right $\mathrm{S} 1$ radiculopathy. The patient underwent a L5 laminectomy for resection of the intradural tumor. The pathological specimen was diagnosed as GBM and the tumor cells stained positive for GFAP, S100 and vimentin (Fig. 2). This unusual diagnosis prompted an MRI of the brain, which revealed a ring-enhancing mass in the left mediotemporal lobe, measuring $2.5 \times 2.8 \times 2.4 \mathrm{~cm}$, and an 8-mm nodular focus of enhancement adjacent to the left inferior frontal gyrus (Fig. 3). An MRI scan of the cervical and thoracic spine was also performed, revealing posterior arachnoid thickening at the C6-7 level, which was consistent with leptomeningeal spread of the disease. The patient was scheduled to receive 46 Gy whole-brain radiation in 2-Gy daily fractions, 46 Gy cervical spine radiation in 2-Gy daily fractions and 5,000 cGy sacral radiation in 200-cGy daily fractions. Although the patient tolerated treatment well, with improved headaches and back pain after receiving a total of $49 \mathrm{~Gy}$, he decided to discontinue treatment. The patient subsequently exhibited increased fatigue, worsening low back pain and an abrupt decline in functional status. Despite being encouraged to complete the radiation therapy and receiving information on available home-health psychiatric services, the patient declined further treatment and was transferred to hospice 3 months after the initial diagnosis. The patient succumbed to the disease 3 months later, 6 months after the initial diagnosis.

\section{Discussion}

Extracranial metastasis of GBM is extremely rare, occurring in $<2 \%$ of patients. When either extra- or intracranial metastasis occurs, the median time from initial tumor diagnosis to recognition of metastases is 8.5 months (9). Several theories on why GBM is rarely associated with extracranial metastasis have been proposed. According to one of those theories, due to the highly aggressive behavior of GBMs, patients succumb to their intracranial disease before there is sufficient time for distant metastasis to develop. The majority of the patients succumb to GBM within 20 months, usually secondary to intracranial mass effect or invasion, sometimes with elevation 



Figure 2. Case 1: Needle biopsy of the lung demonstrated effacement of alveolar spaces by tumor on (A) hematoxylin and eosin (H\&E) staining (magnification, x100) and (B) nests of metastatic glioma separated by alveolar septae on immunohistochemical staining for glial fibrillary acidic protein (GFAP) (magnification, $\mathrm{x} 400)$. Case 2: Needle biopsy of the neck mass demonstrates tumor cells resembling the patient's original malignant glioma on (C) H\&E staining (magnification, $\mathrm{x} 200$ ) and (D) glial differentiation within the tumor on GFAP immunohistochemical staining (magnification, x200). Case 3: Permanent surgical section of the intradural lumbosacral mass confirmed histopathological characteristics of glioblastoma on (E) H\&E staining (magnification, $\mathrm{x} 400$ ) and (F) strong diffuse immunoreactivity for GFAP on GFAP immunohistochemical staining (magnification, x100), where the negatively stained areas consist of blood vessels exhibiting exuberant microvascular proliferation.

of the intracranial pressure. Since metastasis is rare, systemic staging or screening with body CT, MRI, or positron emission tomography imaging is not standard practice. Therefore, metastasis may occur more frequently than what is reported in the literature, but is not clinically recognized prior to fatal brain herniation. In fact, there are two reports of metastatic GBM following bilateral lung transplantation from a donor with GBM, highlighting that there may be unrecognized GBM micrometastases at the time of death $(10,11)$.

Another theory suggest that there are unique barriers in the brain that prevent hematogenous and lymphatic spread, including the dura and the thickened basement membrane of the blood vessels. As a result, most cases of metastasis are considered to occur following craniotomy or intraventricular shunt placement, during which time tumor cells gain access to the blood stream through defects in the meningeal and parenchymal blood vessels that are created from such manipulation (12). Stereotactic biopsies may also cause disease spread, most likely through seeding of tumor cells during the invasive procedure $(1,13,14)$. In cases with scalp metastasis in close proximity to the craniotomy or biopsy site, it is difficult to establish whether it is a result of true blood-born metastasis or seeding of tumor cells. However, Armstrong et al (15) recently reported a case of a scalp metastasis circumscribed within the soft tissue, several centimeters away from the craniotomy site, where neither an indwelling catheter or an established breach of the dura was present. Although cases 1 and 2 (metastases to the lung and posterior neck, respectively) occurred months after craniotomy, these metastatic sites are more distant compared to the scalp and local cranial vault tissues and, thus, are more likely attributable to blood-born metastasis rather than local tumor seeding at the time of the initial craniotomy. 


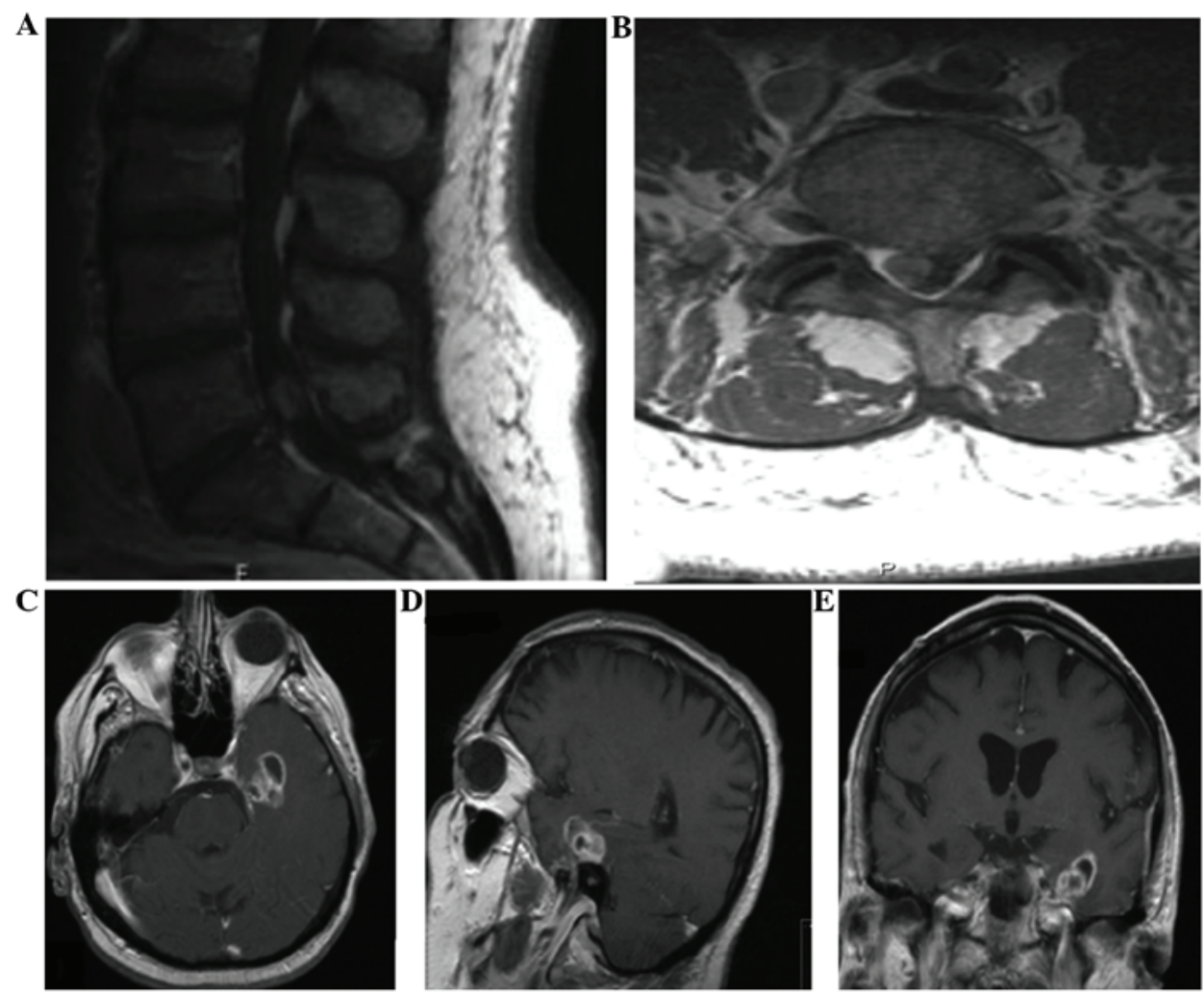

Figure 3. Case 3: Contrast-enhanced magnetic resonance imaging (MRI) of the (A) lumbar spine sagittal and (B) axial sequences reveal intradural spinal metastasis of glioblastoma to the L5-S1 region. Contrast-enhanced MRI of the (C) brain axial, (D) sagittal and (E) coronal sequences show the primary intracranial glioblastoma that was discovered subsequent to the metastatic intradural lumbar spinal lesion.

Mentrikoski et al (12) proposed that another reason primary brain tumors rarely metastasize outside of the CNS is the lack of extracellular matrix protein. In other organs, tumors metastasize by first invading through connective tissue, basement membranes and blood vessels. It is hypothesized that, as there is a near absence of collagen and fibronectin within the CNS, malignant CNS tumor cells lack a component necessary for metastasizing outside the CNS. However, fibronectin may very well be present in GBM cells, as demonstrated in the following study: Using reverse transcription-polymerase chain reaction, immunocytochemistry and enzyme-linked immunosorbent assay, Lin et al (16) demonstrated that the expression of vascular endothelium-derived fibronectin was more prominent in endothelial cells of high-grade gliomas compared to those of low-grade gliomas. The authors also demonstrated that the interaction of glioma cells and vascular endothelial cells in vitro induced fibronectin release from vascular endothelial cells, which in turn stimulated glioma cell migration. This migration was inhibited by fibronectin-blocking antibody. These observations challenge the popular view that there is a near absence of collagen and fibronectin within the CNS and explain how endothelial cells in GBMs have the ability to invade through connective tissue and subsequently metastasize distally.

The rarity of GBM growing outside the CNS was also investigated by Mourad et al (17). The authors implanted brain tumor cells characterized with respect to in vitro and in vivo morphology, growth rate, anchorage-independent growth, GFAP expression and cytogenetic analysis and major histocompatibility complex typing. Intracerebral and systemic GBM growth was assayed in three different rodent models with increasingly different immunological variants between the implanted cells and the murine host, an isograft, an allograft and a xenograft. The authors demonstrated growth of intracerebral and systemic tumors in isografts, growth of intracerebral tumors and suppression of systemic tumors in allografts and lack of growth of intracerebral and systemic tumors in xenografts. The results indicated that tumor cells implanted outside the CNS were able to form tumors, unless there was a significant difference between the immunotype of the implanted cells and the host, suggesting the role of the immune response in controlling these neoplasms and supporting that there are physical and systemic barriers that impede systemic GBM metastasis. GBMs appear to be antigenic -not immunogenic- and, therefore, should be amenable to targeted immunotherapy.

Another study by Park et al (18) proposed that the emergence of neoplastic subclones may be responsible for GBM metastasis. In 6 patients with GBM metastases, primary and metastatic tumor tissue was evaluated for common genetic alterations found in GBMs, such as TP53 mutations, CDKN2A/p16 deletions, epidermal growth factor receptor amplifications and allelic losses of chromosomes 1p, 10q and 19q. In 2 of these patients, different molecular genetic patterns were observed between the primary and metastatic lesions, primarily differences in TP53 mutations between the CNS lesion and the metastases or among the metastases themselves. This finding suggests that certain metastatic lesions may be 
characterized by TP53 mutations and represent the emergence of subclones that were not necessarily dominant in the primary tumor.

Unlike GBMs, gliosarcomas, a rare variant of grade IV gliomas, have a tendency to metastasize. Consideration of the histological characteristics of these tumors may indicate the possible mechanisms underlying GBM metastasis. Treatment for GBM, particularly irradiation, may cause sarcomatous metaplasia of glial cells and help GBMs acquire the necessary extracellular matrix proteins for vascular invasion and hematogenous dissemination to distant extracranial sites. Gliosarcomas consist of an admixture of gliomatous and sarcomatous components and are more likely to invade connective tissue. Thus, these tumors exhibit a greater propensity for extracranial metastasis. For a long time, gliosarcoma was considered to be a GBM, in which the vascular elements had become sarcomatous. Recent molecular studies, however, have refuted this theory and proposed that the sarcomatous component arises from metaplastic transformation of glial cells rather than vascular elements. Beaumont et al (19) reviewed these molecular characteristics, which include identical p53 and PTEN mutations, p16 deletions and amplification of MDM2 and CDK4. Common cytogenetic abnormalities have also been demonstrated in the two lineages, including trisomy 7, deletion of 9p, monosomy 10 and 17 and non-random chromosome $\mathrm{X}$ inactivation. Although sarcomatous metaplasia appears to be a possible mechanism of extracranial metastasis for GBMs, sarcomatous components were not identified on histological review of our three cases.

Spinal metastasis of GBM, in contrast to extra-axial metastases, is more common, has been extensively described in autopsy series and is considered to occur through either leptomeningeal or intramedullary dissemination. Symptomatic spinal metastases largely occur in relatively younger patients with a longer duration of survival. However, upon diagnosis of such a metastasis the prognosis is dismal, with a mean subsequent survival time of 2.8 months and poor response of the metastatic disease to radiation therapy (20-22). CSF cytology is poorly sensitive for spinal metastasis; however, GFAP expression appears to be a histological marker for potential spinal spread. Patients with GFAP-negative tumors, representing the presence of poorly differentiated astrocytic glioma cells, exhibit aggressive CSF dissemination with little infiltration at the primary site, whereas the opposite is observed for GFAP-positive, highly differentiated tumor cells $(23,24)$. Case 3 described in our series was characterized by spinal metastasis; however, it should be noted that the patient initially presented with radicular symptoms prior to the diagnosis of the primary brain tumor; furthermore, he was aged $>60$ years and his tumor was GFAP-positive. However, the survival time from diagnosis to death was the poorest of all three patients, which is consistent with the poor prognosis of GBM spinal metastases reported in the literature. The treatment of patients with spinal GBM metastases remains challenging, as there are few precedents in the clinical literature. Available data suggest chemotherapy, spinal irradiation and palliative resection, although the prognosis remains poor (25).

The standard treatment for intracranial GBM is maximal safe surgical resection, followed by concurrent radiotherapy and chemotherapy (26). However, clinical studies evaluating different treatment strategies for metastatic disease are sparse and the optimal treatment for metastatic disease has not been determined. From our case series and from the review of current literature, two patterns of GBM distal metastasis emerge, namely neuroaxial and systemic metastasis. In patients with neuroaxial dissemination, there may be a role for debulking surgery and irradiation. Our patient in case 3 received craniospinal radiation following initial resection of the spinal metastasis. By contrast, for patients with systemic distal metastasis, organ-specific considerations should be made when planning adjuvant therapy (27). The phenomenon of an intracerebral tumor continuing to grow while the same tumor diminishes in response to systemic chemotherapy has been well described in animal and human models (28). In terms of definitive oncological treatment for metastatic GBM, our case series and other reports reviewed here suggest that treatment should focus on systemic chemotherapy. Lun et al (9) conducted a meta-analysis of 88 cases of extracranial metastasis of GBM and found that patients treated with surgery + radiation + chemotherapy + cerebrospinal shunting exhibited the longest average survival interval from metastasis to death compared to those treated with surgery alone, radiation alone, surgery + radiation, or surgery + radiation + chemotherapy.

The optimal chemotherapeutic agents, however, have yet to be defined. The most commonly used treatments include temozolomide and nitrosurea-based agents (26,29-31). Methylguanine-DNA methyltransferase tumor status and higher levels of expression in tumors may confer resistance to alkylating agents (32). Bevacizumab, a vascular endothelial growth factor A-binding antibody, has also been used as a monotherapy or in combination with chemotherapy $(33,34)$. However, recent randomized controlled studies in the United States and Europe have demonstrated the lack of efficacy of bevacizumab $(35,36)$. Our patient in case 1 with GBM metastasis to the lung received treatment with bevacizumab in combination with carboplatin. The combination of multiple resections, systemic chemotherapy, bevacizumab and ventriculoperitoneal shunting in this patient resulted in 13 months of survival from metastasis to death - the longest reported survival of lung metastases from GBM to date. Lun et al (9) found that lung metastasis is a statistically significant prognostic factor of poor outcomes, with a relative decrease of 2.7 months in the interval from detection of extracranial metastases to death. From our experience with this case, it appears that bevacizumab may be a valuable adjunct in this subset of patients, who have traditionally had an extremely poor prognosis.

Recent reports help explain why bevacizumab may not be effective in arresting disease progression. As explained above, the invasive quality of GBM is partly mediated by vascular proliferation. The abnormal endothelium of GBM that allows this proliferation and, possibly, vascular invasion and subsequent distant metastasis, was recently described by Wang et al (37), who identified stem-like cells in GBM tumors by expression of CD133. A subset of these cells expresses vascular endothelial cadherins, CD144. The authors demonstrated that when $\mathrm{CD} 133^{+} / 144^{+}$(double-positive cells) were cultured in endothelial medium, they differentiated into endothelial cells with a downregulation of CD144 and upregulation of endothelial markers, including CD105, which were capable 
of forming vascular networks within abnormal, thickened channels. In addition, when $\mathrm{CD} 133^{+} / \mathrm{CD} 144^{-}$cancer stem cells were co-cultured with GBM tumor cells, some of the CD133 ${ }^{+}$CD144- cells converted to double-positive cells. Both double-positive and $\mathrm{CD} 133^{+} / \mathrm{CD} 144^{-}$cells resulted in highly infiltrative tumors when implanted in the striatum of immunodeficient mice. The investigators then tested the effects of bevacizumab and N-[N-(3,5-difluorophenacteyl)-L-alanyl]S-phenylglycine t-butyl ester, a $\gamma$-secretase inhibitor that inhibits Notch signaling, on the conversion of CD133 ${ }^{+} / \mathrm{CD} 144$ to double-positive cells and the conversion of double-positive cells to $\mathrm{CD} 105^{+}$cells. Bevacizumab did not block the conversion of $\mathrm{CD} 133^{+} / \mathrm{CD} 144^{-}$to double-positive cells, but did prevent double-positive cells from differentiating into endothelial cells $\left(\mathrm{CD} 105^{+}\right)$. On the other hand, N-[N-(3,5-difluorophenacteyl)-L -alanyl]-S-phenylglycine t-butyl ester had the opposite effect; it blocked the conversion of $\mathrm{CD} 33^{+} / 144^{-}$to double-positive cells but did not affect further maturation, explaining the inefficacy of bevacizumab alone in the treatment of GBM and suggesting that combination therapy with both agents may be the optimal treatment.

In conclusion, extracranial metastasis of GBM is a rare phenomenon and the published literature regarding this disease entity is sparse. The role of molecular and genetic factors in metastatic GBM may have implications in terms of therapeutic targets and adjuvant treatments; however, more thorough genetic profiling in larger case series is mandated. The interactions of glioma cells and their vascular niche is an additional component of GBM invasion and metastasis to consider, as is the presence of extracellular matrix proteins that emerge as a result of this interaction. As our therapeutic armamentarium for intracranial GBMs continues to evolve, along with a better understanding of their biology, and as we are able to prolong the survival of patients with such tumors, the number of cases of metastatic GBMs may continue to increase. In this study, we elucidated the role of systemic chemotherapy with temozolamamide \pm bevacizumab in the treatment of metastatic GBMs; however, the role of staging for prognosis and specific treatment paradigms must be further defined.

\section{References}

1. Saad AG, Sachs J, Turner CD, et al: Extracranial metastases of glioblastoma in a child: case report and review of the literature. J Pediatr Hematol Oncol 29: 190-194, 2007.

2. Rajagopalan V, El Kamar FG, Thayaparan R and Grossbard ML: Bone marrow metastases from glioblastoma multiforme - a case report and review of the literature. J Neurooncol 72: 157-161, 2005.

3. Datta CK, Weinstein JD, Bland JE, Brager PM and Stewart MA: A case of cervical lymph node metastasis resulting from glioblastoma multiforme. W V Med J 94: 276-278, 1998.

4. Fecteau AH, Penn I and Hanto DW: Peritoneal metastasis of intracranial glioblastoma via a ventriculoperitoneal shunt preventing organ retrieval: case report and review of the literature. Clin Transplant 12: 348-350, 1998.

5. Piccirilli M, Brunetto GM, Rocchi G, Giangaspero F and Salvati M: Extra central nervous system metastases from cerebral glioblastoma multiforme in elderly patients. Clinico-pathological remarks on our series of seven cases and critical review of the literature. Tumori 94: 40-51, 2008.

6. Templeton A, Hofer S, Töpfer M, et al: Extraneural spread of glioblastoma - report of two cases. Onkologie 31: 192-194, 2008.

7. Widjaja A, Mix H, Gölkel C, et al: Uncommon metastasis of a glioblastoma multiforme in liver and spleen. Digestion 61: 219-222, 2000
8. Yasuhara T, Tamiya T, Meguro T, et al: Glioblastoma with metastasis to spleen - case report. Neurol Med Chir (Tokyo) 43: 452-456, 2003.

9. Lun M, Lok E, Gautam S, Wu E and Wong ET: The natural history of extracranial metastasis from glioblastoma multiforme. J Neurooncol 105: 261-273, 2011.

10. Armanios MY, Grossman SA, Yang SC, White B, Perry A, Burger PC and Orens JB: Transmission of glioblastoma multiforme following bilateral lung transplantation from an affected donor: case study and review of the literature. Neuro Oncol 6: 259-263, 2004.

11. Chen H, Shah AS, Girgis RE and Grossman SA: Transmission of glioblastoma multiforme after bilateral lung transplantation. J Clin Oncol 26: 3284-3285, 2008.

12. Mentrikoski M, Johnson MD, Korones DN and Scott GA: Glioblastoma multiforme in skin: a report of 2 cases and review of the literature. Am J Dermatopathol 30: 381-384, 2008.

13. Bouillot-Eimer S, Loiseau H and Vital A: Subcutaneous tumoral seeding from a glioblastoma following stereotactic biopsy: case report and review of the literature. Clin Neuropathol 24: 247-251, 2005.

14. Sloan AE, Ahluwalia MS, Valerio-Pascua J, et al: Results of the NeuroBlate System first-in-humans phase I clinical trial for recurrent glioblastoma: clinical article. J Neurosurg 118: 1202-1219, 2013.

15. Armstrong TS, Prabhu S, Aldape K, et al: A case of soft tissue metastasis from glioblastoma and review of the literature. J Neurooncol 103: 167-172, 2011.

16. Lin ZX, Yang LJ, Huang Q and Fu J: Activated vascular endothelia regulate invasion of glioma cells through expression of fibronectin. Chin Med J (Engl) 123: 1754-1761, 2010.

17. Mourad PD, Farrell L, Stamps LD, Chicoine MR and Silbergeld DL: Why are systemic glioblastoma metastases rare? Systemic and cerebral growth of mouse glioblastoma. Surg Neurol 63: 511-519, 2005.

18. Park CC, Hartmann C, Folkerth R, et al: Systemic metastasis in glioblastoma may represent the emergence of neoplastic subclones. J Neuropathol Exp Neurol 59: 1044-1050, 2000.

19. Beaumont TL, Kupsky WJ, Barger GR and Sloan AE: Gliosarcoma with multiple extracranial metastases: case report and review of the literature. J Neurooncol 83: 39-46, 2007.

20. Maslehaty H, Cordovi S and Hefti M: Symptomatic spinal metastases of intracranial glioblastoma: clinical characteristics and pathomechanism relating to GFAP expression. J Neurooncol 101: 329-333, 2011.

21. Medhkour A and Chan M: Extremely rare glioblastoma multiforme of the conus medullaris with holocord and brain stem metastases, leading to cranial nerve deficit and respiratory failure: a case report and review of the literature. Surg Neurol 63: 576-583, 2005.

22. Vertosick FT Jr and Selker RG: Brain stem and spinal metastases of supratentorial glioblastoma multiforme: a clinical series. Neurosurgery 27: 516-522, 1990.

23. Arita N, Taneda M and Hayakawa T: Leptomeningeal dissemination of malignant gliomas. Incidence, diagnosis and outcome. Acta Neurochir (Wien) 126: 84-92, 1994.

24. Onda K, Tanaka R, Takahashi H, Takeda N and Ikuta F: Cerebral glioblastoma with cerebrospinal fluid dissemination: a clinicopathological study of 14 cases examined by complete autopsy. Neurosurgery 25: 533-540, 1989.

25. Hübner F, Braun V and Richter HP: Case reports of symptomatic metastases in four patients with primary intracranial gliomas. Acta Neurochir (Wien) 143: 25-29, 2001.

26. Stupp R, Mason WP, van den Bent MJ, et al: Radiotherapy plus concomitant and adjuvant temozolomide for glioblastoma. N Engl J Med 352: 987-996, 2005.

27. Astner ST, Pihusch R, Nieder C, et al: Extensive local and systemic therapy in extraneural metastasized glioblastoma multiforme. Anticancer Res 26: 4917-4920, 2006.

28. Steinbok P, Dolman CL and Goldie JH: Variation in response to CCNU of glioblastoma multiforme in brain and cervical lymph node. Case report. J Neurosurg 62: 918-921, 1985.

29. Brandes AA, Tosoni A, Cavallo G, et al; GICNO: Temozolomide 3 weeks on and 1 week off as first-line therapy for recurrent glioblastoma: phase II study from Gruppo Italiano Cooperativo di Neuro-Oncologia (GICNO). Br J Cancer 95: 1155-1160, 2006.

30. Gaviani P, Silvani A, Lamperti E, Botturi A, Fariselli L, Simonetti G, Ferrari D and Salmaggi A: Rechallenge with temozolomide in recurrent glioma. Neurol Sci 32: S247-S249, 2011. 
31. Perry JR, Rizek P, Cashman R, Morrison M and Morrison T: Temozolomide rechallenge in recurrent malignant glioma by using a continuous temozolomide schedule: the 'rescue' approach. Cancer 113: 2152-2157, 2008.

32. Hegi ME, Diserens AC, Gorlia T, et al: MGMT gene silencing and benefit from temozolomide in glioblastoma. N Eng J Med 352: 997-1003, 2005.

33. Chamberlain MC and Johnston SK: Salvage therapy with single agent bevacizumab for recurrent glioblastoma. J Neurooncol 96: 259-269, 2010.

34. Friedman HS, Prados MD, Wen PY, et al: Bevacizumab alone and in combination with irinotecan in recurrent glioblastoma. J Clin Oncol 27: 4733-4740, 2009.
35. Chinot OL, Wick W, Mason W, et al: Bevacizumab plus radiotherapy-temozolomide for newly diagnosed glioblastoma. N Engl J Med 370: 709-722, 2014

36. Gilbert MR, Dignam JJ, Armstrong TS, et al: A randomized trial of bevacizumab for newly diagnosed glioblastoma. N Engl J Med 370: 699-708, 2014.

37. Wang R, Chadalavada K, Wilshire J, et al: Glioblastoma stem-like cells give rise to tumour endothelium. Nature 468: 829-833, 2010. 